\title{
CUIDAR POR MEIO DA EDUCAÇÃO: A EXTENSÃO UNIVERSITÁRIA E A PROMOÇÃO DA SAÚDE DE ADOLESCENTES E JOVENS
}

\author{
Carla Cardi Nepomuceno ${ }^{1}$ \\ Ana Carolina de Oliveira Jerônymo ${ }^{1}$ \\ Betânia Maria Fernandes ${ }^{2}$ \\ Sandra Carvalho de Freitas ${ }^{3}$
}

\begin{abstract}
RESUMO: Este trabalho trata-se de um relato de experiência decorrente do projeto de extensão intitulado "Prevenindo a gravidez não planejada e o contágio de infecções sexualmente transmissíveis/HIV/AIDS com adolescentes e jovens". O projeto é realizado, desde 2010, em uma escola da rede estadual de ensino de um município da Zona da Mata Mineira, por acadêmicos de Enfermagem, e atende estudantes na faixa etária entre 15 e 21 anos, e adota um referencial que envolve a integração de saberes à luz da teoria freiriana, o que possibilita estabelecer uma parceria significativa. Tem como objetivos dialogar e esboçar conhecimentos que discorram sobre como escolher e usar os métodos anticonceptivos e, também, enfatizar a importância do conhecimento das formas de prevenção do contágio de infecções sexualmente transmissíveis e HIV/AIDS. Nesse projeto, foi possível constatar, por meio do relato de seus participantes, o estabelecimento de um maior diálogo com os pais e o uso consciente dos métodos anticoncepcionais, além da percepção de professores do maior desenvolvimento do conhecimento dos adolescentes e jovens sobre a sexualidade. Assim, na educação, é imprescindível a construção de vínculo entre os participantes do processo de ensino-aprendizagem, através de uma metodologia participativa, dinâmica, criativa e de forma contínua para atingir os objetivos pré-estabelecidos.
\end{abstract}

PALAVRAS-CHAVE: Saúde do adolescente. Sexualidade. Enfermagem. Educação em saúde.

\section{Taking care through education: university extension and the promotion of health of adolescents and youngsters}

\begin{abstract}
This work is based on an experience report result of the university extension project entitled "Preventing unwanted pregnancy and the contagion of sexually transmitted infections (STI)/HIV/AIDS of adolescent and youngsters". This project has been occurring since 2010 at a state school in a city of Minas Gerais Forest Zone by academics of Nursing Course and it assists students aged between 15 and 21 years old, adopting a referential that involves the integration of knowledge under Freire's theory, making possible to establish a significant partnership, since the activities are structured according to the reality and the necessities of the target audience. This work aims to dialogue with and to outline knowledge that discourses about how to choose and use contraceptive methods, and also emphasizing the importance of knowing the ways of preventing from the contagion of sexually transmitted infections and HIV/AIDS. During this project, it was established through the report of its participants a broader dialogue with parents

\footnotetext{
${ }^{1}$ Acadêmicas do curso de Enfermagem da Universidade Federal de Juiz de Fora (carlinhanepo@hotmail.com; akarololiveiraj@hotmail.com).

${ }^{2}$ Doutora em Enfermagem pela Universidade Federal do Rio de Janeiro, professora adjunta na Faculdade de Enfermagem da Universidade

Federal de Juiz de Fora (betaniafernandes@uol.com.br).

${ }^{3}$ Mestranda em Enfermagem pela Faculdade de Enfermagem da Universidade Federal de Juiz de Fora (samdrinh@hotmail.com).
} 
and the conscious use of contraceptive methods, and it was perceived, by teachers, a broader development of adolescent's knowledge about sexuality. Thus, in education, it is essential building a bond between the participants of teaching-learning process, through a participative, dynamic, creative and continuous methodology, in order to achieve the pre-aimed goals.

KEYWORDS: Adolescent health. Sexuality. Nursing. Health education.

\section{INTRODUÇÃO}

A extensão universitária é a principal forma de articulação entre a Universidade e setores externos da sociedade, sendo redimensionada com ênfase na relação entre teoria e prática, na perspectiva de uma relação dialógica baseada na troca de saberes (JEZINE, 2004). Essa integração do meio acadêmico com a comunidade permite estreitar os laços e, além disso, oportuniza a elaboração de trabalhos científicos.

A atividade de extensão é entendida como um processo articulador, que oportuniza aos docentes enriquecer e instrumentalizar os conhecimentos adquiridos pelas experiências práticas. Esse processo vertical permite que ocorra a troca de saberes, tendo como objetivos a produção de conhecimentos resultantes das realidades brasileira e regional, a reconstrução e redefinição de conceitos estabelecidos, a garantia da participação efetiva da comunidade e da atuação da universidade nesta e vice-versa (SOUZA et al., 2004).

No contexto da graduação em Enfermagem, a inserção nas atividades de extensão como dispositivo de ensino contribui de maneira significativa na formação acadêmica, uma vez que os alunos inseridos nesse processo educativo constroem conhecimentos e vivenciam situações singulares. Nesse sentido, desde 2010 vimos tendo a experiência no desenvolvimento de um projeto intitulado "Prevenindo a gravidez não planejada e o contágio de Infecções Sexualmente Transmissíveis/HIV/AIDS com adolescentes e jovens" em uma escola da rede estadual de ensino de um município da Zona da Mata Mineira.

Ao entender que o saber não é absoluto e que está sempre sendo construído e reconstruído, somos facilitadores do processo de ensino e aprendizagem, utilizando-nos de uma metodologia para abordar temáticas que vão além de teorias das salas de aula, pois estão presentes na vida cotidiana, corroborando Paulo Freire quando afirma que:

A educação tem caráter permanente. Não há seres educados e não educados. Estamos todos nos educando. Existem graus de educação, mas estes não são absolutos (FREIRE, 2006, p. 28).

Nesse projeto de extensão, utiliza-se a educação em saúde, em consonância com Catrib (2003), com o intuito de contemplar os princípios do Sistema Único de Saúde (SUS) por intermédio da promoção da saúde que, por sua vez, tem por objetivos proporcionar mudanças de comportamento nos indivíduos inseridos em um contexto social, político e econômico. Assim, a educação é uma 
das quatro competências gerais do cuidado do enfermeiro e se enquadra no desafio de realizar ações de educação em saúde a todas as faixas etárias.

O projeto citado é direcionado a adolescentes e jovens, por estarem estes nas fases da vida que envolve profundas mudanças biopsicossociais, especialmente relacionadas à maturação sexual, à busca da identidade adulta e à autonomização frente aos pais, familiares e amigos (SANTOS et al., 2010).

Segundo a Organização Mundial da Saúde (OMS) (1995), a adolescência é a fase correspondente à faixa dos 10 aos 19 anos, sendo a pré-adolescência dos 10 aos 14 anos e a adolescência, propriamente dita, dos 15 aos 19 anos. Esta faixa etária é marcada por um período de transformações físicas e psicológicas; assim, o adolescente apresenta atitudes e comportamentos peculiares nessa faixa de idade, dentre os quais se destaca o desenvolvimento intenso da sexualidade, muitas vezes sem o uso adequado dos métodos anticonceptivos e relações sexuais desprotegidas, podendo acarretar uma gravidez não planejada e o contágio de infecções sexualmente transmissíveis (ISTs) e HIV/ AIDS, além do adiamento de projetos futuros.

Atualmente, a primeira relação sexual ocorre cada vez mais precocemente, pois, segundo dados da OMS, publicados no Programa Nacional de Saúde do Escolar (2009), 22\% dos adolescentes haviam iniciado atividade sexual aos 15 anos de idade. No Brasil, segundo Pantoja (2003), a primeira experiência sexual para as meninas ocorre entre 12 e 15 anos e, para os meninos, entre 11 e 13 anos. Um dos fatores que justificam essa precocidade é a diminuição gradativa da idade média de entrada na puberdade, ou seja, o desenvolvimento fisiológico está antecedendo o cognitivo e o emocional (TRAJMAM et al., 2003).

Outro diagnóstico importante quanto à saúde dos jovens refere-se à divulgação pelo Ministério da Saúde, em 2011, do último boletim epidemiológico relativo à Síndrome da Imunodeficiência Humana Adquirida (AIDS), em que foram notificados 34.218 casos de ISTs e constatou-se uma taxa de incidência da AIDS, no Brasil, de 17,9 casos por 100 mil habitantes. Em 2010, a relação foi de 1,7 caso em homens para cada 1 caso em mulheres. A faixa etária em que a AIDS é mais incidente, em ambos os sexos, é a de 25 a 49 anos de idade (BRASIL, 2011).

A ocorrência de contágio de ISTs ocorre, inúmeras vezes, devido a não utilização correta do método anticonceptivo, à falta de informação e à dificuldade de acesso a eles (SOARES et al., 2008).

Diante do exposto, surgiu a necessidade de implantar o referido projeto de extensão em uma escola, apoiada nos parâmetros curriculares nacionais (PCN) do ensino médio do Brasil que preveem em seus objetivos tornar a aprendizagem escolar sistematizada, consciente e deliberada de modo que o tema "sexualidade" seja trabalhado nas escolas, a fim de que os adolescentes e jovens entendam a dinâmica do funcionamento do próprio corpo e, a partir disso, sejam conscientes ao realizarem decisões pessoais, tais como: não usar drogas, não consumir gorduras e exercer a sexualidade com responsabilidade (BRASIL, 2000).

A Lei de Diretrizes e Bases da Educação Nacional (LDB), como uma norma que auxilia na preparação das atividades a serem desenvolvidas, sugere que os adolescentes e jovens "aprendam a fazer do prazer, do entretenimento, da sexualidade, um exercício de liberdade responsável" 
(BRASIL, 2000, p. 63), promovendo assim o direito de livre escolha e de acesso à informação de forma clara e sem constrangimentos.

Portanto, este relato de experiência resulta da execução do projeto de extensão, financiado pela Próreitoria de Extensão da Universidade Federal de Juiz de Fora (UFJF) a partir da realização de atividades educativas sobre temas relacionados à sexualidade, tendo o objetivo de relatar e refletir sobre a experiência dos discentes de enfermagem ao coordenar os grupos para adolescentes e jovens na escola.

\section{DESCRIÇÃO DAS ATIVIDADES}

Desde 2010, o projeto confere oportunidades aos acadêmicos de ampliarem seus conhecimentos e desenvolverem habilidades. Em 2011, porém, a equipe foi reformulada e o projeto reestruturado para serem realizados sete encontros por turma, em uma escola da rede estadual de ensino do município da Zona da Mata Mineira.

No primeiro semestre de atuação, a ação do grupo era realizada quinzenalmente, mas foi observado que isso dificultava o processo de ensino e aprendizagem. Assim, decidiu-se pela realização semanal, com duração de 40 minutos por semana.

As atividades do projeto iniciaram-se em junho de 2011, atendendo, até então, quatro turmas, sendo três do período noturno e uma do diurno. Até o momento, noventa e dois alunos na faixa etária entre 15 e 21 anos foram contemplados com as atividades do projeto.

A coordenadora, em meio ao aparato teórico e científico, estimula os discentes do projeto a estudarem e pesquisarem sobre as temáticas abordadas. Os acadêmicos, orientados pela coordenadora e por dois professores da escola na qual o projeto é desenvolvido, elaboram um planejamento de atividades com a finalidade de promover a organização para que ocorra o alcance dos objetivos e metas propostas a cada encontro.

Nesse contexto, o ato de planejar pode ser entendido como a elaboração de estratégias a partir de um conjunto de conhecimentos teóricos, científicos e práticos construídos de forma coordenada e direcionada para que elas sejam executadas a fim de alcançar objetivos e metas estabelecidas de maneira sistematizada.

O planejamento do grupo é composto de sete encontros, que têm como primeiro tema abordado questões relacionadas à sexualidade, gênero e direitos sexuais e reprodutivos. Como fundamentação teórica, considera-se que a sexualidade não representa apenas o ato sexual, ela envolve emoções, sentidos, atitudes, o contato visual, físico e afetivo, expresso pelo ser humano em sua magnitude e complexidade. Além disso, o gênero é abordado como um processo de construção social, histórica e cultural das representações do masculino e feminino na prática social (JEOLÁS; FERRARI, 2003). Com a finalidade de tornar mais palpável essa temática repleta de mitos e tabus, estimula-se a fala de experiências e, ao final, utiliza-se um vídeo ${ }^{4}$ para encerrar o encontro.

\footnotetext{
${ }^{4}$ Vídeos disponíveis na internet utilizados na dinâmica do primeiro encontro: "Medo de quê?" e "Acorda, Raimundo, acorda!".
} 
Nosegundoencontroéabordadootemadatomadadedecisões, afimdeaproximarosadolescentesejovens da sua realidade e estimulá-los, por meio de situações cotidianas, a tomarem decisões mais conscientes.

Após a discussão desses conceitos fundamentais, aborda-se a anatomia e fisiologia do sistema reprodutor masculino e feminino, com o auxílio de material educativo (bonecos de papel masculino e feminino). Esse terceiro encontro é fundamental para construir conhecimentos que facilitem a compreensão dos métodos anticoncepcionais.

Noquarto quinto encontros abordam-setodos os métodos anticoncepcionais, divididos, didaticamente, em cincogrupos: comportamentais, hormonais, de barreira, cirúrgicos eo dispositivo intrauterino(DIU).

No sexto encontro é debatido o tema das infecções sexualmente transmissíveis (ISTs) e da síndrome da imunodeficiência adquirida (HIV/AIDS). Como recurso educativo, utilizam-se slides com imagens de diversas patologias transmitidas pelo sexo, com o principal objetivo de incentivar o uso do preservativo masculino em todas as relações sexuais e destacar os principais sinais e sintomas do exposto, para que compreendam a importância de procurar um serviço de saúde no momento adequado.

O último encontro é reservado para abordagem de alguma temática solicitada pelos alunos, que não constava no planejamento. Com a realização desses grupos, em quatro turmas, foram discutidos temas sobre o aborto, a violência sexual e drogas. Também, nesse dia, realiza-se uma dinâmica de encerramento e aplica-se um questionário de avaliação.

Em cada encontro educativo, um acadêmico é responsável por elaborar um relatório do grupo e a avaliação das atividades feita pelos estudantes. O termo "avaliação", aqui enquadrado, relacionase a perceptiva teórica de Andrighetto e Ricther (2009, p. 1545), que diz:

[...] Assim, notoriamente a avaliação não pode limitar-se aos conhecimentos transmitidos pelo docente e adquiridos pelo aluno, mas sim, a implementação da soma das experiências do meio escolar com o meio sociocultural trazido pelo aluno, tendo como resultado deste processo a contínua expansão do saber. Pois... "A mente que se abre a uma nova ideia jamais voltará ao seu tamanho original" (Albert Einstein).

\section{AVALIAÇÃO DAS ATIVIDADES}

Conforme Junqueira e colaboradores (2011), a educação em saúde é uma proposta inovadora que utiliza uma metodologia participativa e reflexiva, contribuindo para a obtenção de melhores hábitos de vida. Nesse conceito, a vida saudável, incluindo o desenvolvimento da sexualidade, não é entendida apenas pelo desenvolvimento adequado do corpo, mas como o inter-relacionamento entre melhores condições de moradia, trabalho, alimentação, educação, serviços de saúde, lazer, enfim, melhores condições de vida da comunidade. 
A educação em saúde deve abranger todos os aspectos constituintes de determinada comunidade, articulando o emocional com o biológico, pois é impossível descrever a sexualidade sem vinculála ao relacionamento entre pais e filhos, ou, ainda, explicar sobre os métodos anticonceptivos sem considerar os sentimentos presentes em uma relação afetiva.

Com o decorrer das atividades do projeto, os alunos tornaram-se mais participativos, expondo suas dúvidas e debatendo os temas propostos. Isso permitiu o direcionamento das discussões, de acordo com suas demandas, para o uso de preservativo masculino e feminino e outros métodos anticonceptivos, além da anticoncepção de emergência, uma vez que muitos adolescentes e jovens afirmaram usar este último frequentemente, comprometendo a sua saúde sexual, devido à ingestão de doses inadequadas de hormônios. Os alunos também demonstraram não ter conhecimento sobre o uso correto, sua ação e a interação dos métodos anticoncepcionais com outros medicamentos ou mesmo com outros métodos, demonstrando curiosidade sobre o assunto abordado, principalmente sobre o anticoncepcional oral, o diafragma e o preservativo feminino.

Em relação à sexualidade e aos direitos sexuais e reprodutivos, foi observado, por meio da fala dos alunos, o desconhecimento sobre tais temáticas, relacionando a sexualidade apenas ao ato sexual e não a um conjunto de aspectos referentes a sentimentos e demonstrações do mesmo. Muitos participantes ou quase que a totalidade desses desconhece os direitos sexuais e reprodutivos relativos à oferta dos métodos anticoncepcionais e a sua disponibilidade na Unidade de Atenção Primária à Saúde (UAPS). Os adolescentes afirmaram, ainda, nunca terem recebido informações sobre a relevância da consulta de controle do câncer do colo do útero e da mama e desconhecerem a importância de sua realização quando se tem uma vida sexualmente ativa.

As temáticas foram abordadas de forma interativa, o que oportunizou aos graduandos de Enfermagem o exercício da capacidade de coordenar e mediar o conhecimento de todos participantes do grupo e vivenciar a experiência da prática pedagógica pela troca de saberes durante os encontros, desenvolvendo habilidades como o falar em público, o pensar crítica e reflexivamente e ter iniciativa e flexibilidade perante as adversidades.

No desenvolvimento das atividades educativas deste projeto de extensão, foi expressiva a participação dos alunos nos encontros, sendo baixa a porcentagem de faltosos. Além disso, os professores da escola, responsáveis pela disciplina de Biologia, nos auxiliaram nos encontros, sendo os agentes motivadores dos adolescentes e jovens. Assim, acreditamos que essa parceria facilitou o envolvimento dos estudantes com as atividades dos encontros, como também possibilitou um amadurecimento dos mesmos sobre os aspectos que envolvem o universo da sexualidade.

Ressaltamos, como ganho para o projeto, seu patenteamento junto à secretaria de educação do município pelos professores da escola, valorizando e subsidiando a importância do mesmo para os alunos. Esta integração, também, fez com que professores de outras escolas do município se interessassem pelas atividades oferecidas pelo projeto de extensão.

Como resultado positivo, além dos que já descrevemos até o momento, está o despertar do interesse dos alunos após a participação nas atividades do projeto, principalmente com relação a temáticas que envolvam o ciclo menstrual, o período reprodutivo e a sua ligação com a reprodução 
masculina e feminina. Com isso, ressalta-se a importância de esse tema ser abordado com maior intensidade, o que possibilitaria, ao final, verificar que os adolescentes e jovens terão construído conhecimentos de acordo com a sua realidade social e cultural.

Consideramos que os encontros foram produtivos devido à intensidade das discussões levantadas e às dúvidas apresentadas, além dos momentos de intervalo em que muitos alunos procuraram os acadêmicos para esclarecer dúvidas sobre os temas trabalhados, o que demonstrou entrosamento entre a turma e os facilitadores do processo de ensino e aprendizagem.

Como resultados imediatos deste projeto de extensão destacam-se, por meio da fala dos alunos participantes, o estabelecimento de um diálogo mais aberto com os seus pais e o desenvolvimento de uma maior responsabilidade, evidenciada pelo aumento do uso correto dos métodos anticonceptivos, com destaque para o preservativo masculino (a dinâmica na qual os alunos são convidados a colocar o preservativo masculino no protótipo peniano evidenciou que muitos dos participantes não sabiam a forma correta de utilizá-lo). Em relação aos resultados mediatos, espera-se ampliar o número de escolas para a realização de encontros educativos baseados no contexto social, econômico e cultural, de modo que esta ação auxilie na diminuição do índice de gravidez não planejada e do contágio de IST/HIV/AIDS entre os adolescentes e jovens atendidos pelas atividades do projeto.

\section{CONSIDERAÇÕES FINAIS}

Para trabalhar efetivamente com a educação em saúde para os adolescentes e jovens é preciso determinar objetivos que vão além de transmitir informações para promover a sua saúde. Essa ação pretende, também, oferecer subsídios para que eles tenham pensamento crítico e vivam sua sexualidade de forma segura e orientada.

Nesse sentido, as atividades desenvolvidas pelo projeto alcançaram, por meio das dinâmicas com recursos metodológicos, a participação ativa dos alunos no processo de construção de conhecimentos, reformulação de conceitos e ideias, reflexão sobre os contextos de vida e época em que estão inseridos e exercício de uma prática sexual consciente.

Assim espera-se, com a realização periódica dos grupos educativos com adolescentes e jovens, que haja colaboração para a redução do índice de gravidez não planejada e o contágio ISTs/HIV/ AIDS, bem como estimulação da prática do sexo seguro com o uso de preservativo. Além disso, têm-se como intuito a promoção da aproximação dos adolescentes e jovens da unidade de saúde e a sensibilização para que recorram ao SUS para a aquisição de métodos anticonceptivos, assim como para a prevenção do câncer cérvico-uterino e de mama. 


\section{REFERÊNCIAS}

ANDRIGHETTO, M. J.; RICTHER, C. J. Avaliação escolar. In: Programa de Pós- Graduação em Ensino de Ciência e Tecnologia,2009. Paraná, Anais... Paraná:PPGECT. Disponível em: $<\mathrm{http} / / \mathrm{www}$. pg.utfpr.edu.br/sinect/anais/artigos/13\%20Formacaodeprofessoresnoensinodecienciaetecnologia/ Formacaodeprofessoresnoensinodecienciaetecnologia_artigo3.pdf >. Acesso em: 12 mar. 2012.

BRASIL. Ministério da Educação. Parâmetros Curriculares Nacionais Ensino Médio. Brasília, 2000. Disponível em: <http://portal.saude.gov.br/portal/saude/visualizar_texto. cfm?idtxt=33728\&janela=1>. Acesso em: 6 abr. 2012.

. Ministério da Saúde. Boletim Epidemiológico DST/AIDS. Brasília, 2011. Disponível em: $\overline{\mathrm{http}} / / \mathrm{www} . a i d s . g o v . b r / s i t e s / d e f a u l t / f i l e s / a n e x o s /$ publicacao/2011/50652/boletim_aids_2011_ final_m_pdf_26659.pdf. Acesso em: 6 abr. 2012

CATRIB, A. M. F. Promoção da saúde: saber fazer em construção. In: Barroso, G. T.; Vieira, N. F. C; Varela, Z. M. V. Educação em saúde no contexto da promoção humana. Fortaleza: Edições Demócrito Rocha, 2003.

FREIRE, Paulo. Pedagogia da Autonomia: saberes necessários à prática educativa. 34. ed. São Paulo: Paz e Terra, 2006.

IBGE, Instituto Brasileiro de Geografia e Estatística. Pesquisa Nacional da Saúde do Escolar. Rio de Janeiro, 2009. Disponível em: < http://www.ibge.gov.br/home/estatistica/populacao/pense/ pense.pdf>. Acesso em: 3 mar. 2012.

JEOLÁS, L. S; FERRARI, R. A. P. Oficinas de prevenção em um serviço de saúde para adolescentes: espaço de reflexão e de conhecimento compartilhado. Ciência \& Saúde Coletiva, Rio de Janeiro, v. 8, n. 2, p. 611-620, 2003. Disponível em: <http://www.scielo.br/scieloOrg/php/reflinks. php? refpid $=$ S1413-8123200900030003000017\&lng=en\&pid $=$ S1413-81232009000300030 > . Acesso em: 23 abr. 2012.

JEZINE, E. As práticas curriculares da extensão universitária. In: CONGRESSO BRASILEIRO DE EXTENSÃO UNIVERSITÁRIA, 2., 2004, Belo Horizonte. Anais... Brasília: UNESCO, 2004.

JUNQUEIRA, M. A. B. et al. Educação que produz saúde: relato de uma experiência. Em Extensão, Uberlândia, v. 10, n. 2, p. 156-161, jul./dez, 2011. Disponível em: <http://www. revistadeextensao.proex.ufu.br/viewarticle.php?id=495>. Acesso em: 23 maio. 2012.

ORGANIZAÇÃO MUNDIAL DE SAÚDE. La salud de los jóvenes: un reto y una esperanza. OMS: Genebra, 1995. 120p. Disponível em: <http://www.scielo.br/scieloOrg/php/reflinks. php?refpid=S0104-1169200000020000500019\&lng=en\&pid=S0104-11692000000200005>. Acesso em: 10 abr. 2012.

PANTOJA, A. L. N. "Ser alguém na vida": uma análise sócio-antropológica da gravidez/ maternidade na adolescência em Belém do Pará, Brasil. Cad. Saúde Pública, Rio de Janeiro, v. 2, n. 19, p. 334-335, out. 2003. Disponível em: <http://www.scielo.br/pdf/csp/v19s2/a15v19s2. pdf>. Acesso em: 22 mar. 2012. 
SANTOS, E. C. et al. Gravidez na adolescência: análise contextual de risco e proteção. Psicologia em Estudo, Maringá, v. 15, n. 1, p. 73-85, jan.-mar, 2010. Disponível em: <http://www.scielo. br/scielo.php?script=sci_arttext\&pid=S1413-73722010000100009>. Acesso em: 10 mar. 2012.

SOARES, S. M. et al. Oficinas sobre sexualidade na adolescência: revelando vozes, desvelando olhares de estudantes do ensino médio. Revista de Enfermagem da Escola Anna Nery, Rio de Janeiro, v. 12, n. 3, p. 485-491, set. 2008. Disponível em: <http://dx.doi.org/10.1590/S141481452008000300014>. Acesso em: 18 mar. 2012.

SOUZA, A. A. et al. Projeto de extensão na formação da enfermagem. Revista Eletrônica de Extensão. Florianópolis, v.1, n.1, p. 1-8, dez. 2004. Disponível em: <http://www.periodicos. ufsc.br/index.php/extensio/article/view/1445/4509>. Acesso em: 12 jan. 2012.

TRAJMAN A. et al. Knowledge about STD/AIDS and sexual behavior among high school students in Rio de Janeiro, Brazil. Cad. Saúde Pública, Rio de Janeiro, v. 19, n. 1, p. 127133, 2003. Disponível em: <http://www.scielo.br/scieloOrg/php/reflinks.php?refpid=S14138123200900030003000018\&lng=en\&pid=S1413-81232009000300030 . Acesso em: 14 abr. 2012. 